\title{
TANDEM-L: \\ A MISSION PROPOSAL FOR MONITORING DYNAMIC EARTH PROCESSES
}

\author{
Alberto Moreira ${ }^{(1)}$, Gerhard Krieger ${ }^{(1)}$ Marwan Younis $^{(1)}$, Irena Hajnsek $^{(1)}$, Kostas \\ Papathanassiou $^{(1)}$, Michael Eineder ${ }^{(2)}$, Francesco De Zan ${ }^{(1)}$
} (1) Microwaves and Radar Institute, German Aerospace Center (DLR), 82234 Oberpfaffenhofen,
Germany, e-mail: alberto.moreira@dlr.de

\author{
(2) Remote Sensing Technology Institute, German Aerospace Center (DLR), \\ 82234 Oberpfaffenhofen, Germany
}

\begin{abstract}
Tandem- $\mathrm{L}$ is a mission proposal for an innovative interferometric L-band radar instrument that enables the systematic monitoring of dynamic Earth processes using advanced techniques and technologies. The mission is science driven aiming to provide a unique data set for climate and environmental research, geodynamics, hydrology and oceanography. Important application examples are global forest height and biomass inventories, measurements of Earth deformation due to tectonic processes and/or anthropogenic factors, observations of ice/glacier velocity field and 3-D structure changes, and the monitoring of soil moisture and ocean surface currents. The Tandem-L mission concept consists of two cooperating satellites flying in close formation. The Pol-InSAR and repeat-pass acquisition modes provide a unique data source to observe, analyse and quantify a wide range of mutually interacting processes in the bio-, litho-, hydro- and cryosphere. The systematic observation of these processes benefits from the high data acquisition capacity and the novel high-resolution wide-swath SAR imaging modes that combine digital beamforming with a large reflector antenna. This paper provides an overview of the Tandem-L mission concept and its main application areas. It is planned to realise the Tandem- $\mathrm{L}$ mission in cooperation with NASA/JPL. The mission concept was developed in detail in a joint two-year pre-phase A study and it will be further studied in the next 18 months. This will allow a cost-effective implementation, whereby each partner contributes its predevelopments and experience. According to current planning, the Tandem-L satellites could be launched in 2019.
\end{abstract}

\section{INTRODUCTION}

Tandem- $\mathrm{L}$ is a proposal for an interferometric and polarimetric SAR mission at L-band for mapping dynamic Earth processes. The motivation for this mission proposal comes from the increasing science requirements for a continuous and global monitoring of climate and environmental variables with high resolution and on a reliable way. Examples of the essential variables to be measured by Tandem-L in a systematic way are [3], [10]:

- Above ground forest biomass and its 3-D vertical structure distribution. Observation of changes in forest height and biomass (e.g. due to deforestation or afforestation), changes in biodiversity, etc.

- Earth surface deformation (e.g. due to seismic movements, volcano eruptions, land slides, subsidence, uplift, etc.).

- Retreat and accumulation in ice and snow covered regions, velocity field estimation of land ice movement within a high velocity range variation.

- Changes in surface soil moisture and land use (high resolution maps).

- Measurements of ocean surface currents.

In order to achieve the ultimate goal to estimate these essential variables in a systematic and reliable way, the following requirements are posed on the SAR mission concept:

- Single-pass interferometry for estimation of forest height, biomass and 3-D structure by means of multibaseline Pol-InSAR.

- Repeat-pass acquisition mode for estimation of surface deformation with differential interferometry.

- L-Band as the most appropriate frequency for Pol-InSAR and D-InSAR acquisition modes due to the following reasons:

- High bandwidth of the available frequency allocation (85 MHz).

- Penetration capability in vegetated areas allowing the forest biomass estimation up to 500 tons/ha.

- High coherence values in repeat-pass mode.

- Low RF interference and ionospheric perturbations when compared to lower frequency bands. 
- Short revisit cycle for small temporal decorrelation.

- High data rate acquisition and downlink capability to allow a systematic acquisition of dynamic processes (acquired data volume larger than 1-2 TB/day).

In section 3 the measurement and mission concept will be presented based on these requirements. Due to the need of having Pol-InSAR and D-InSAR modes in the mission concept, one satellite should have a nearly circular and polar orbit within a narrow tube of a few hundred meters for D-InSAR operation, while the second satellite should have an orbit which provides the required baselines for Pol-InSAR. The same helix orbit concept as in the TanDEM-X mission is adopted for the second satellite where a small eccentricity offset and a shift of the ascending node allows for a flexible adjustment of the desired baselines [1].

\section{SCIENCE JUSTIFICATION}

The estimation of dynamic processes on Earth surfaces requires systematic, long term and continuous observation strategies in order to detect short and long term changes with a sufficient accuracy. Depending on the environmental and/or anthropogenic process to be observed there is a need for having different time intervals in the acquisition plan.

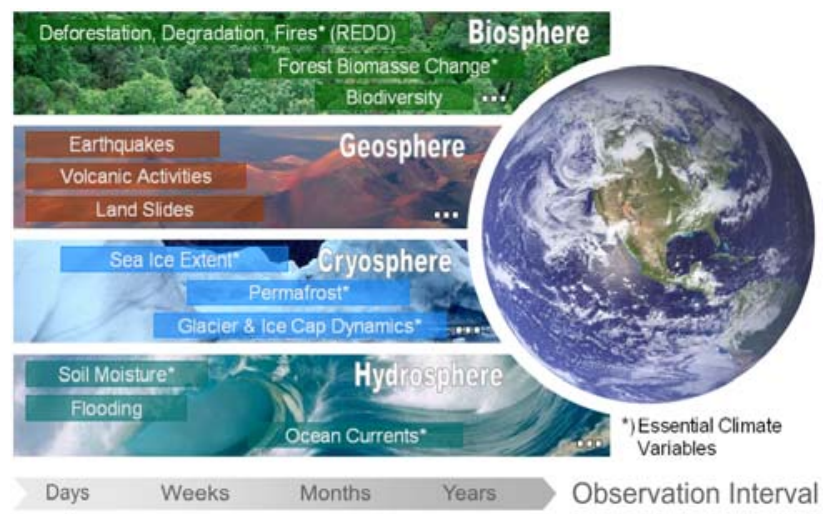

Figure 1: Dynamics of different Earth spheres and the requested interval for sampling the corresponding process in an unambiguous way

Existing SAR sensors could already demonstrate that radar plays an important role in the parameter estimation related to essential environmental, climate and anthropogenic processes. Today, SAR sensors are mostly covering only small and selected areas and do not acquire data in a long term and systematic way in order to make reliable statements about Earth's processes changes. In some cases large areas are being acquired, but the required sampling interval as shown in Fig. 1 is not fulfilled.

One important feature of Tandem- $\mathrm{L}$ is therefore to achieve observation intervals of weeks to several months on a global scale with an appropriate sampling to characterise dynamic processes. A second important feature is a systematic acquisition strategy with a global coverage that allows generating consistent time series over 5 years of mission lifetime. The combination of short observation intervals and systematic data acquisition will enable frequent observations as required for the monitoring of seismic movements as well as the observation of long term processes with seasonal and/or yearly cycles like the growth of vegetation.

The main scientific focus of Tandem- $\mathrm{L}$ is placed on two application areas, the biosphere and geosphere. However, with the requirements for a systematic and global mapping with short repeat times Tandem-L will be also very useful for other application areas in hydro- and cryosphere. The Tandem-L mission will provide a new insight and will increase the information content in bioand geo-sciences and represents therefore a unique concept that will provide a step forward towards a holistic view of global land processes.

Tandem- $\mathrm{L}$ will be the first mission that will measure biomass with an accuracy of $20 \%$ on a global scale and in addition estimate its yearly change throughout the mission lifetime. With this information the uncertainty in the terrestrial stored above ground biomass will be drastically reduced. Biomass will be derived from the direct estimate of the forest height and 3-D forest structure that will be measured by Tandem-L [8], [9].

\section{MEASUREMENT CONCEPT}

For the data acquisition an innovative concept has been worked out. The satellite system as it is planned up to now will operate in two basic data acquisition modes:

1) The 3-D structure mode employs fully-polarimetric single-pass SAR interferometry (Pol-InSAR) to acquire structural parameters and quasi-tomographic images of volume scatterers like vegetation, sand, and ice.

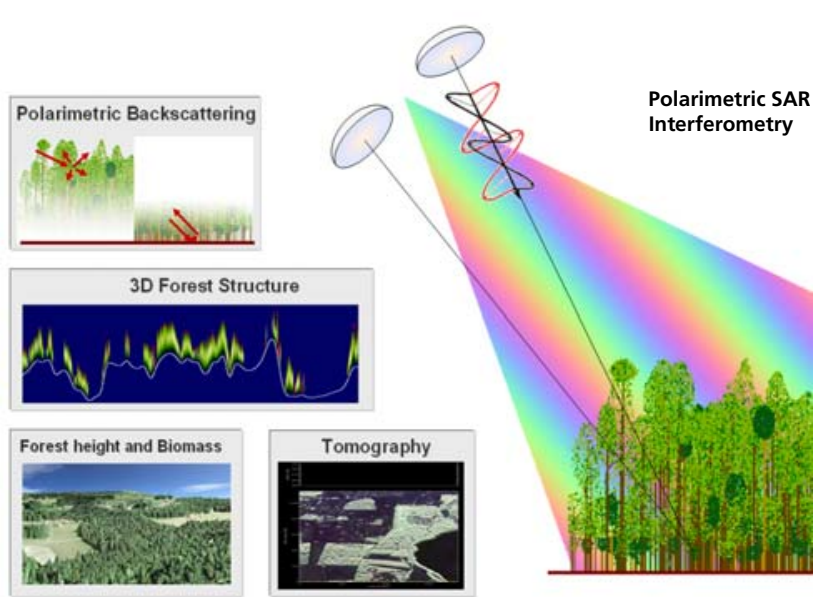

Figure 2: 3-D structure mode using single-pass SAR interferometry 
2) The deformation mode employs repeat-pass interferometry (InSAR) in an ultra-wide swath mode in order to measure small shifts on the Earth surface with millimeter accuracy in short repetition intervals.

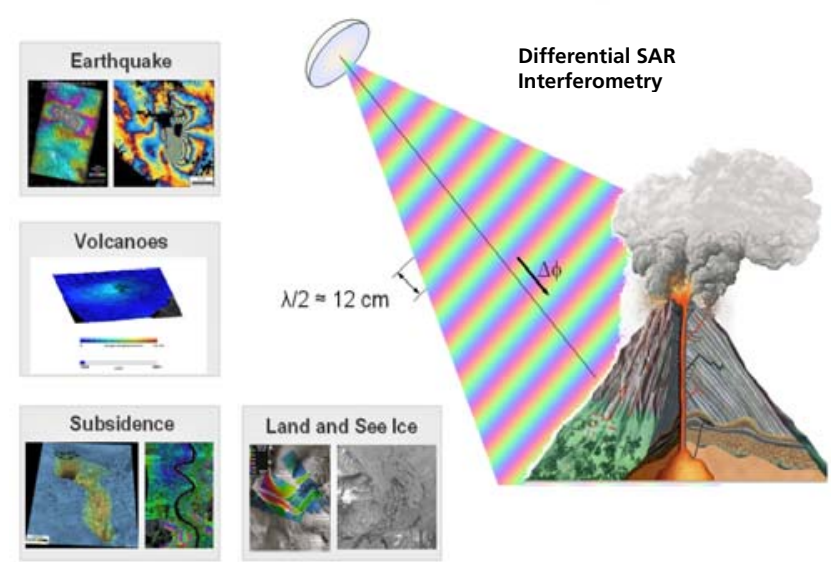

Figure 3: Deformation mode using repeat-pass SAR interferometry

Both acquisition modes enable a systematic and global mapping of the Earth with a high spatial resolution due to the use of digital beamforming techniques. The deformation mode requests a short orbital repeat cycle, whereas the 3-D structure mode needs only a repeat pass time of one month due to slower changes in the vegetation cover.

In case of critical events and hazards (earthquakes, flooding, volcano eruption, dike breaks etc.) it will be possible to adapt the satellite acquisition plan to a certain area of interest that can then typically be mapped within one or two days.

The deformation mode can be operated in any position of the orbit cycle, while the 3-D structure mode requires adequate interferometric baselines that are only present at specific latitudes depending on the adjusted satellite formation parameters. Hybrid operation modes in connection with digital beamforming will allow resolving the conflicts in the acquisition mode due to different user requirements [5], [6], [7].

\section{DIGITAL BEAMFORMING}

One key technology of Tandem- $\mathrm{L}$ is the use of a large reflector antenna and the use of a digital feed array. While all feed elements are used during transmission, allowing the illumination of a large image swath, 2-3 feed elements are activated at one time during the receive window. The active feed element positions are periodically shifted in synchrony with the systematic variation of the direction of arrival from the swath echoes. The advantages of this concept are manifold. First, the use of a large reflector antenna in connection with digital beamforming allows the reduction of the transmit power by a factor of 3-4 in comparison to the traditional SAR concept for the same imaging parameters. Second, it allows the mapping of a much wider swath (ca. $350 \mathrm{~km}$ ) in high resolution stripmap mode with low range ambiguity levels [2], [4], [5]. The fully polarimetric acquisition in stripmap mode with a wide swath is possible without the constraints of conventional SAR systems. This leads, however, to a large data rate and requires the implementation of advanced technologies for high data rate downlink. The downlink requirements can however be alleviated by employing new hybrid SAR modes suggested in [5]. These modes enable variable resolutions within a single scene and allow by this for an optimum adaptation to the nonhomogeneous resolution requirements from the different science disciplines.

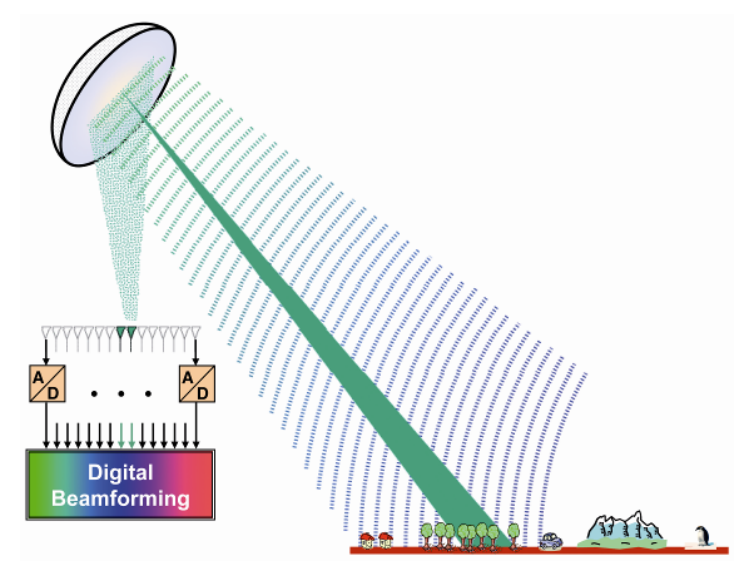

Figure 4: Concept of digital beamforming for high resolution, wide-swath radar systems

\section{IMPLEMENTATION PLAN}

One most challenging task in the Tandem- $\mathrm{L}$ realisation is the development of two identical satellites with a cost effective implementation approach and at the same time a high performance in order to fulfil the demanding scientific needs.

The joint pre-phase study with NASA/JPL has been started in 2008 and completed in October 2010. The study has demonstrated a concept for a joint realisation of a Tandem-L/DESDynI type of mission. An extension of this study will be carried out until the end of 2012 in order to optimize the work sharing for the spacecraft and instrument development and to explore further possibilities for a low-cost realization approach. Currently, the mission proposal is scheduled for a mission operation time of five years with a launch in 2019. At the German side the decision for the realisation of the Tandem- $\mathrm{L}$ mission is depending on the approval of the necessary funding which is expected for the end of 2012. 


\section{SUMMARY}

Tandem- $\mathrm{L}$ is an innovative mission proposal for mapping Earth dynamics with an unprecedented accuracy and capability. Advanced measurement techniques like singlepass Pol-InSAR allow the estimation of new essential environmental and climate variables. The instrument concept includes the innovative technology of digital beamforming in connection with a large reflector antenna which allows fulfilling the so far contradicting user requirements for a fully polarimetric imaging mode with wide swath and high resolution.

Table 1: Tandem-L Time Schedule

\begin{tabular}{|l|l|}
\hline Year & Planning \\
\hline 2011-2012 & $\begin{array}{l}\text { Extended Pre-Phase A } \\
\text { \& Technology Studies }\end{array}$ \\
\hline 2013 & Phase A (1 Year) \\
\hline $2014-2015$ & Phase B (1 Year) \\
\hline $2015-2019$ & Phase C/D (4 Years) \\
\hline 2019 & Launch of Satellites \\
\hline $2019-2024$ & Nominal Mission Duration \\
\hline
\end{tabular}

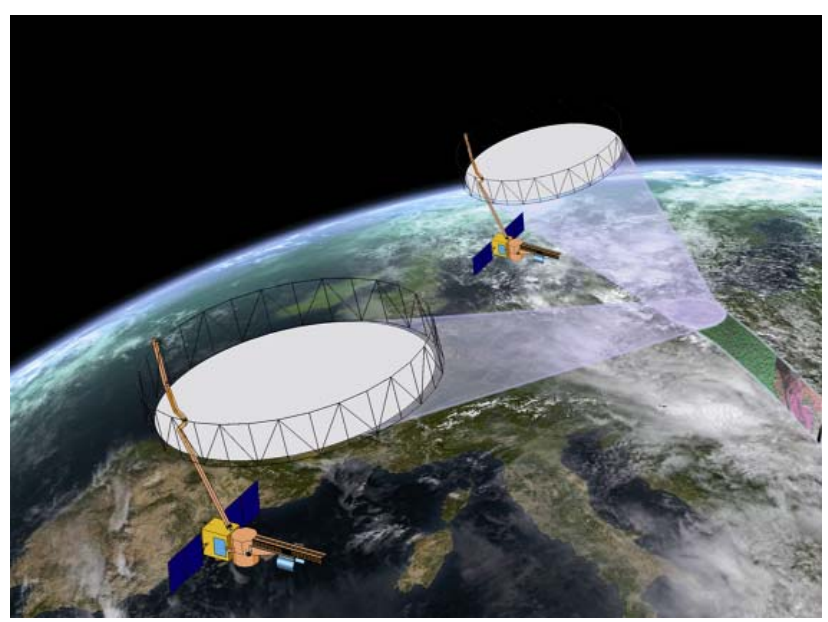

Figure 5: Artistic view of a Tandem-L formation with two satellites (preliminary design achieved during a joint DLR and NASA/JPL pre-phase A study)

In addition to the science objectives described in this paper, Tandem-L has the potential for allowing many other applications and products due to its global and systematic acquisition plan. As an example, single-pass Pol-InSAR can also be used to estimate the bare soil topography as complementary information to the surface DEM that will be provided with the TanDEM-X mission with 12 meter sampling and with a height accuracy of 2 meters.

\section{ACKNOWLEDGEMENT}

We would like to thank the Tandem-L science and project team for their contributions to the pre-phase A study. In addition, we would like also to thank the DESDynI science team for sharing their expertise in ecology and geo-sciences and the NASA/JPL research/engineering team for the constructive discussions.

\section{REFERENCES}

[1] Krieger, Gerhard; Moreira, Alberto; Fiedler, Hauke; Hajnsek, Irena; Werner, Marian; Younis, Marwan; Zink, Manfred: TanDEM-X: A Satellite Formation for High Resolution SAR Interferometry. IEEE Transactions on Geoscience and Remote Sensing, 45 (11), 2007, pp. 3317 -3341 .

[2] Krieger, Gerhard; Gebert, Nicolas; Moreira, Alberto: Multidimensional Waveform Encoding: A New Digital Beamforming Technique for Synthetic Aperture Radar Remote Sensing. IEEE Transactions on Geoscience and Remote Sensing, 46 (1), 2008, pp. 31 - 46.

[3] IPCC 2007 - IPCC Fourth Assessment Report: Climate Change, Intergovernmental Panel on Climate Change, http://www.ipcc.ch

[4] Freeman, A. et al.: DESDynI - A NASA Mission for Ecosystems, Solid Earth, and Cryosphere Science. Proceedings of the Pol-InSAR Workshop, Frascati, January 26-30, 2009.

[5] Krieger, G. et al.: The Tandem-L Mission Proposal: Monitoring Earth's Dynamics with High-Resolution SAR Interferometry. Proceedings of the IEEE Radar Conference, Pasadena, May 4-8, 2009.

[6] Krieger, G., Hajnsek, I., Papathanassiou, K. P., Younis, M., Moreira, A.: Interferometric Synthetic Aperture Radar (SAR) Missions Employing Formation Flying. Proceedings of the IEEE, vol. 98, no. 5, May 2010, pp. 816-843.

[7] Moreira, A., Krieger, G., Hajnsek, I., Papathanassiou, K., Eineder, M., De Zan, F., Younis, M. and Werner, M.: Tandem-L: Monitoring the Earth's Dynamics with InSAR and Pol-InSAR. In Proc. PolInSAR Workshop, Frascati, Italy (ESA SP-668), January 2009.

[8] Cloude, S.R., and Papathanassiou, K .P., 1998, "Polarimetric SAR Interferometry", IEEE Transactions on Geoscience and Remote Sensing, vol. 36, no. 5, 1998, pp. 1551-1565.

[9] Reigber, A., and Moreira, A.: First Demonstration of Airborne SAR Tomography Using Multibaseline L-Band Data. IEEE Transactions on Geoscience and Remote Sensing, vol. 38, no. 5, 2000, pp. 2142-2152.

[10] The Changing Earth. New Scientific Challenges for ESA's Living Planet Programme. ESA SP-1304, 2006. 